Meta

Journal des traducteurs

Translators' Journal

\title{
La musique du mot et du concept, ou certains problèmes de traduction poétique
}

\section{Roland Maisonneuve}

Volume 23, numéro 1, mars 1978

La traduction poétique

URI : https://id.erudit.org/iderudit/003542ar

DOI : https://doi.org/10.7202/003542ar

Aller au sommaire du numéro

Éditeur(s)

Les Presses de l'Université de Montréal

ISSN

0026-0452 (imprimé)

1492-1421 (numérique)

Découvrir la revue

Citer cet article

Maisonneuve, R. (1978). La musique du mot et du concept, ou certains problèmes de traduction poétique. Meta, 23(1), 73-85.

https://doi.org/10.7202/003542ar d'utilisation que vous pouvez consulter en ligne. 


\section{La musique du mot et du concept, ou certains problèmes de traduction poétique}

I

Tout poème est un univers : « univers d'univers d'univers », dit un sage hindou; univers d'éléments imperceptibles que le poète veut rendre perceptibles, par toute une musique d'images, de sons, de rythmes, de jaillissements d'idées, de chocs de concepts.

Voyez cette sculpture africaine, qui vient du Mali. Elle montre cinq fils de fer tordus. Qu'est-ce? les prémisses de l'art abstrait? Non, un danseur... Et quel danseur! Les fils de fer semblent partir dans toutes les directions. Le danseur, c'est l'univers ; l'univers animé, propulsé, vivifié par l'Esprit invisible qui danse de joie. Ainsi en est-il du poème, pour le poète : ce dernier frémit, en lui-même, d'une certaine musique, faite de l'exercice de ses yeux, et de ses sens, et de son intelligence, et de ses rêves, de sa vie, et de sa parole. C'est cette musique qui est son poème. C'est cette musique - et non seulement des mots et des rythmes - que le traducteur doit reproduire par un univers verbal.

II n'y a pas de création poétique en général.

Il n'y a pas de traduction poétique en général.

Ill y a des poètes aussi divers que la Rose des vents. L'un veut cerner son soi-mème. L'autre veut parler à tous, et de tous, en parlant de soi. L'un n'a qu'un regard : la nature. L'autre sonde tous les manèges des fêtes foraines de l'amour. Il en est qui veulent dire le monde, ou le révolutionner, ou le transfigurer, ou créer un univers verbal - sorte de paradis artificiel, de drogue dorée - suprême refuge hors d'un monde de néant. D'autres fuient dans l'invisible, cherchent à découvrir ou anéantir leur vrai soi dans l'Essence qui est celle de l'univers, ou dont émane l'univers ; ou encore luttent avec le langage pour exprimer l'indicible de Dieu, ou leur quête de Dieu, leurs combats avec Dieu ou contre Dieu, les tourments de leur chair et de leur esprit.

Et chaque poète est, en soi, tout aussi divers. «Je suis l'insaisissable multiple », répète un griot africain, en s'accompagnant de divers instruments : sa voix pourrait être celle de tout poète. 
C'est dire immédiatement la gageure qu'est la traduction, et les épreuves qui attendent le traducteur.

Certes il lui faut d'abord bien savoir jouer de son propre clavier, c'est-àdire être maitre de son propre langage, en connaître et expérimenter les richesses, les subtilités, les possibilités. Il lui faut certes - tel le joueur de marionnettes qui étreint son gantelet - saisir le rythme, les sonorités, les saveurs verbales de la langue qu'il traduit. Mais s'il ne revit pas, en lui-même, la musique intérieure des textes qu'il a devant lui, sa traduction sera formelle et bulle d'air.

Baudelaire connaissait imparfaitement l'anglais, mais sa traduction de Poe est admirable : infidèle quelquefois par le vocable, mais incantatoire, et de l'incantation même de l'original.

Le traducteur n'est pas seulement en face d'un langage. Il faut qu'empruntant le chemin des mots, il parvienne et s'unisse à l'univers complexe qu'ils signifient.

Il y a là un long voyage en profondeur, durant lequel le traducteur regarde le texte, le contemple, se le dit à lui-même, en soupèse les sonorités, en interroge les rythmes, comme un jeune amoureux qui fait peu à peu connaissance de la personne qu'il aime.

Alors seulement peut-il commencer à traduire valablement.

Le poète, a-t-on dit, est un voyant. Le traducteur doit aussi voir, et par les yeux du poète qu'il traduit.

S'il traduit Isaïe, il doit être - non par imitation servile, mais en esprit souffle de prophète. S'il traduit Abu Sa'id, il doit avoir cervelle de soufi. Pour bien dire Silesius, ne faut-il pas être alchimiste? Pour bien comprendre Rimbaud, il faut être "maître en fantasmagorie "»; pour rendre Lautréamont, jongleur en anarchies.

L'authentique traducteur a donc quelque chose du caméléon. Mais ce n'est pas un phénomène extérieur de mimétisme. Il s'agit de sympathie intérieure, ou d'intuition, ou quête d'une expérience - neuve pour lui - qui le fascine.

C'est pourquoi les traductions qu'on appelle «les belles infidèles » sont finalement décevantes. Elles séduisent le regard, mais elles sont, pour l'affamé, fades comme des potirons.

Les vraies traductions sont celles où le traducteur ne s'impose pas luimême, mais s'efface pour se laisser posséder, pour laisser transparaître l'auteur original. Il y reste son accent, quelque chose de sa propre voix, c'est évident, mais c'est un autre qu'on écoute. Quand Claudel traduit les Joujoux de Coventry Patmore, ce n'est pas Claudel que j'entends, mais, par Claudel, Coventry Patmore.

1. Rimbaud, Une saison en enfer, Guvres complètes, Pléiade, Gallimard, 1946, p. 213. 
THE TOYS

My little Son, who looked from thoughtful eyes, And moved and spoke in quiet grown-up wise, Having my law the seventh time disobeyed, I struck him, and dismissed With hard words and unkissed,

- His Mother, who was patient, being dead.

Then, fearing lest his grief should hinder sleep,

I visited his bed,

But found him slumbering deep,

With darkened eyelids, and their lashes yet

From his late sobbing wet.

And I, with moan,

Kissing away his tears, left others of my own;

For, on a table drawn beside his head,

He had put, within his reach,

A box of counters and a red-veined stone,

A piece of glass abraded by the beach,

And six or seven shells,

A bottle with bluebells,

And two French copper coins, ranged there with careful art,

To comfort his sad heart.

So when that night I prayed

To God, I wept, and said :

$\mathrm{Ah}$, when at last we lie with tranced breath,

Not vexing Thee in death,

And Thou rememberest of what toys

We made our joys,

How weakly understood

Thy great commanded good,

Then fatherly not less

Than I whom Thou hast moulded from the clay,

Thou'lt leave Thy wrath, and say,

"I will be sorry for their childishness. \$

\section{LES JOUJOUX}

Mon petit garçon, dont les yeux ont un regard pensif et qui dans ses mouvements et ses paroles a les manières tranquilles d'une grande personne, ayant désobéi pour la septième fois à ma loi, je le battis et le renvoyai durement sans l'embrasser, sa mère qui était patiente étant morte. Puis, craignant que son chagrin ne l'empêchât de dormir, j'allai le voir dans son lit, où je le trouvai profondément assoupi avec les paupières battues et les cils encore humides de son dernier sanglot. Et je l'embrassai, à la place de ses larmes laissant les miennes. Car sur une table tirée près de sa tête il avait rangé à la portée de sa main une boîte de jetons et un galet à veines rouges, un morceau de verre arrondi trouvé sur la plage, une bouteille avec des campanules et deux sous français, disposés bien soigneusement, pour consoler son triste coeur ! Et cette nuit-là quand je fis à Dieu ma prière je pleurai et je lui dis : " $\mathrm{Ah}$, quand à la fin nous serons là couchés et le souffle suspendu, ne vous causant plus de 
fâcherie dans la mort, et què voús vous souviendrez de quels joujoux nous avons fait nos joies, et combien faiblement nous avons pris votre grand commandement de bonté, alors non moins paternellement que moi que vous avez formé de votre limon, vous laisserez votre colère et vous direz : "J'ai pitié de ces pauvres enfants!»

Traduire, c'est entreprendre un voyage dans l'inccnnu.

Quelquefois une transposition vous vient comme un éclair. Le plus souvent, le traducteur peut dire avec Delacroix : « Je fais, je refais, je recommence, et tout cela n'est point ce que je cherche encore ${ }^{2}$. $\$$

Tout traducteur connaît ce piétinement dont parle Rouault, " atroce même aux très patients artistes, et qui étonne beaucoup ceux qui croient que la vie en art s'obtient facilement avec deux ou trois tons, deux ou trois formes, bavées, crachées, en s'en foutant ${ }^{3} \gg$.

Même la trouvaille qui jaillit en un éclair est fruit d'un labeur silencieux, continu, qui se poursuit dans l'esprit du traducteur sans qu'il en ait conscience. On raconte souvent - avec des variantes chaque fois plus merveilleuses l'histoire de Picasso et de son visiteur américain venu lui acheter un tableau. Picasso n'a rien dont il puisse ou veuille se défaire rapidement. Il rentre dans son atelier, brosse, en quelques coups de pinceatix, une nature morte, revient vers l'américain, et la lui propose : «Ma dernière œuvre. » L'américain s'en va, ravi. Imposture, dira-t-on. Et cependant la toile est aujourd'hui l'un des chefs-d'œuvre d'une galerie privée d'Amérique. Le silencieux labeur continuel est fécond. Picasso livre son secret, lorsqu'il fait cette confidence à un journaliste : «J'avais hâte chaque soir de retourner au travail, je voulais savoir ce qui va se passer ${ }^{4}$.»

S'adressant à un jeune poète, Rilke remarque : «Les œuvres d'art sont d'une infinie solitude.» II ajoute : «Rien n'est pire que la critique pour les aborder. Seul l'amour les peut saisir, les garder, être juste envers elles. » «Les porter jusqu'à son terme, puis enfanter, tout est là ${ }^{5}$. 》

Telle est la clef d'or, pour le traducteur.

II

Traduire un poème, c'est faire jaillir, par certains rythmes, sonorités, vocables d'une langue autre que celle de l'original, un univers identique à celui de l'original, c'est-à-dire un autre poème.

Prenons un texte d'Auden, assez connu : Refugee Blues.

2. Delacroix, Journal, Plon, t. II, 1932, p. 94.

3. G. Rouault-A. Suarès, Correspondance, Arland, Gallimard, 1960, p. 100

4. C. Roy, l'Amour de la peinture, Gallimard, 1956.

5. $\quad$ Rilke, Lettres à un jeune poète, Grasset, 1937, p. 17 et 33-34. 
Say this city has ten million souls,

Some are living in mansions, some are living in holes;

Yet there's no place for us, my dear, yet there's no place for us.

Once we had a country and we thought it fair,

Look in the atlas and you'll find it there :

We cannot go there now, my dear, we cannot go there now.

In the village churchyard there grows an old yew,

Every spring it blossoms anew :

Old passports can't do that, my dear, old passports can't do that.

The consul banged the table and said :

"If you've got no passport, you're officially dead »;

But we are still alive, my dear, but we are still alive..

C'est une sorte de chanson populaire, à la fois désinvolte et désespérée. Ses traducteurs, qui sont poètes, ont surtout joué avec les rythmes.

Paraît que cette ville est de dix millions d'âmes,

Les uns ont de belles maisons, les autres n'ont que des trous :

Hier nous avions une patrie, et hier nous la jugions douce,

Regarde un atlas, et tu la trouveras dedans :

Impossible d'y aller maintenant, ma chère, impossible d'y aller maintenant.

$\mathrm{Au}$ cimetière de mon village là grandit un vieil if, Chaque printemps le voit refleurir :

Les vieux passeports n'en font pas autant, ma chère, les vieux passeports n'en font pas autant.

Le consul frappa sur la table et il dit :

«Si vous n'avez pas de passeport, officiellement vous êtes morts»;

Mais nous sommes encore vivants, ma chère, nous sommes encore vivants... ${ }^{6}$

Le texte est bien cambré, spécialement avec ses ritournelles. Son rythme a cependant une faille : le mot "hier», répété, donne des pieds de plomb à une phrase qui, en anglais, est légère.

L'alchimie des sons est difficile. Chaque terme a sa sonorité. Le vocable original et ses équivalents en traduction ont souvent des univers sonores radicalement différents. Ainsi, dans le Refugee Blues d'Auden, «hole» est, par sa musique, une sorte d'abîme sans fin; « trou » est bref et sec.

Il faut donc, dans la traduction, recomposer un nouvel équilibre musical.

Dans un de ses poèmes - intitulé Song - John Donne murmure, avec une amertume goguenarde, à la femme qu'il aime et qui le fait souffrir :

6. 'Aspects de la littérature anglaise (1918-1940) », Fontaine, no 37, 40, p. 414-416, Alger, 1944. 
When thou sigh'st, thou sigh'st not wind,

But sigh'st my soul away.

Cazamian écrit dans son Anthologie de la poésie anglaise :

Tes soupirs ne sont vent,

Tu soupires mon âme?

Même au prix d'un faible allongement de rythme, ne serait-il pas plus conforme à Donne et à sa musique d'écrire :

Quand tu soupires, ce n'est pas vent que tu soupires,

Mais mon âme que tu expires?

Avançant, avec perplexité, dans le labyrinthe d'une musique étrangère, le traducteur trouve un fil d'Ariane dans l'assonance. Sa propre langue est un immense jardin et jungle de sons, où il peut puiser à volonté, depuis les plus doux et diaphanes jusqu'aux sonorités rocailleuses, abruptes, qui écorchent l'oreille.

Tel poème de Joyce est vraiment poésie pure :

\section{A FLOWER GIVEN TO MY DAUGHTER}

Frail the white rose and frail are

Her hands that gave

Whose soul is sere and paler

Than time's wan wave.

Rosefrail and fair - yet frailest

A wonder wild

In gentle eyes thou veilest,

My blueveined child.

Que donnera ce texte, par l'assonance?

UNE FLEUR OFFERTE À MA FILLE

Frêle la blanche rose et frêles

Les doigts qui l'offrirent

L'âme en est pâle et fanée

Plus que du temps le flot blafard

Fine la rose frêle - mais plus frêle

La sauvage merveille

Qu'en tes beaux yeux tu voiles,

Mon enfant aux veines bleues ${ }^{8}$.

La traduction est une merveille. Il est vrai qu'elle est signée... de Joyce!

7. The Poems of John Donne, edited by Grierson, O.U.P., 1951, p. 18. Pour la traduction,

se rapporter à L. Cazamian, Anthologie de la poésie anglaise, Stock, 1946, p. 12.

8. Aspects de la littérature anglaise $\$, ~ o p$. cit., p. 380 . 
Il y a, par contre, chez Donne, une cacophonie verbale, aride, qui exige une musique à la Stravinski plus qu'à la Schubert.

\section{SONG}

Goe, and catche a falling starre, Get with child a mandrake roote, Tell me, where all past yeares are, Or who cleft the Divels foot,

Teach me to heare mermaides singing, Or to keep off envies stinging, And finde What winde

Serves to advance an honest minde ${ }^{9}$.

Nous proposerions presque ce texte :

\section{CHANSON}

Va chiper une étoile filante, Fais une gosse à la mandragore, Dis-moi où, les années d'antan,

Ou qui fourcha le pied du Diable, Apprends-moi à ouïr le chant des Sirènes,

A parer, de la jalousie, le piquant, Et dénicher Quel vent

Fait aller, de l'avant, un esprit honnête.

Certains poèmes, telle cette chanson de Donne, sont rimés.

La rime multiplie les difficultés... et les défaites du traducteur. Si elle ne s'accompagne d'ailleurs d'un jeu de sonorités et de rythmes, elle est un grelot ridicule qui égrène sa monotonie.

Pourquoi ne pas avoir recours à l'assonance?

Le langage poétique le plus simple est le plus ardu à traduire, surtout si le poème est subtilement construit, et s'il se voile de mystère. Ainsi en est-il des Délires de Sloate, poèmes en prose :

Listening to music, you are a trumpet at one moment, then a clarinet, then one of the solo strings, or another woodwind. It is not a question of hearing, but of being. So that you exist as a drum for a few blank moments and think and act and die as a drum. And so on until the symphony becomes an eternity of lives lived by you in each instrument's particular existence. You loathe, suddenly, the silence, after these lives are over, and a friend's voice calling loudly to you from the next room.

9. The Poems of John Donne, op. cit., p. 8. 
Les mots sont de tous les jours, mais avec une aura d'infini.

Écoutant de la musique, tu es une trompette, un moment, puis une clarinette, puis une des cordes solo, ou un autre instrument à vent. Ce n'est pas une question d'oüe, mais d'être. Ainsi ton existence est-elle celle d'un tambour, pendant quelques moments vides, et tu penses, agis et meurs, comme un tambour. Et ainsi de suite, jusqu'à ce que cette symphonie devienne une éternité de vies vécues, par toi, dans l'existence de chaque instrument en particulier. Tu hais soudain le silence - après que ces vies sont mortes -, et la voix de l'ami qui t'interpelle bruyamment de la pièce voisine.

Ou cet autre poème :

Looking into the young eyes of a child, you spring behind those eyes, and look at yourself with their undeveloped pupils, accustomed only to dolls and toys. Then you jump back to your own head, looking back at the child, and you gasp at the gulf, no wider than a few, unimportant years, but filled with the stagnant and stinking waters of hate, old love, and grief, and oh! so deep, so deep.

C'est un duel entre le silence et la musique.

Fouillant dans les yeux d'un enfant, tu bondis derrière ces yeux, et tu te regardes par leurs pupilles non développées, habituées seulement à des poupées et à des jouets. Alors tu ressautes jusqu'à ta pauvre tête, et tu regardes de nouveau l'enfant, et tu suffoques devant le gouffre, pas plus large que quelques années sans importance, mais rempli d'eaux stagnantes et puantes de haine, vieil amour et chagrin, oh! si profond, profond ${ }^{10}$.

\section{III}

Un problème très particulier est celui des poètes mystiques. Dieu est ineffable. Et ils veulent dire l'ineffable. Ils vivent, dans l'ineffable, une expérience de Dieu particulière. Et ils veulent aussi traduire cet ineffable.

Les traduire tient alors de l'art du funambule.

D'autant que leur expérience s'inscrit dans un univers théologique qu'un simple mot, mal rendu, peut gauchir, défigurer, ou anéantir.

Prenons, par exemple, ce court poème de saint Jean-de-la-Croix : Del Verbo Divino.

Del Verbo divino
la Virgen prenada
viene de camino;
si le dais posada

10. Textes inédits, non inclus dans notre traduction des Délires, parue à la $N . R . F$, en juin 1960, no 90 , p. 1181-1184.

11. Vida e Obras completas de San Juan de la Cruz B.A.C. Madrid 1975, p. 415 Traduction par Benoit Lavaud, dans " Poèmes Mystiques », Cahiers du Rhône, 1942, p. 124-125. 
Le sens est simple : la Vierge est pleine du Verbe de Dieu; elle vient avec lui, sur le chemin qui vient de Dieu et où vous êtes, vivre en votre demeure, c'est-à-dire en vous, si seulement vous ouvrez votre porte et l'accueillez.

Le poète exprime cependant, brièvement et en profondeur, la plus pure théologie de l'Église touchant la Vierge : sa Maternité divine; son union avec son Fils - "Son cour était mon cœur», dit-elle à sainte Brigitte de Suède dans ses Révélations -; sa mission d' "aimant» pour attirer les cours au Christ : Ad Jesum per Mariam; et bien d'autres leçons : la discrétion de Dieu qui s'offre, mais ne s'impose pas; le désir, l'appel, l'abandon, qui ouvrent l'âme à Dieu...

Mais tout cela n'est pas dit, mais suggéré par des vers qui sont comme ces éléments de jeux de construction qui peuvent se combiner de plusieurs manières ; comme autant de flammes qui s'interpénètrent, et défient le traducteur.

Benoît Lavaud, des Frères prêcheurs, propose :

$$
\begin{aligned}
& \text { du Verbe divin } \\
& \text { la Vierge prégnante } \\
& \text { vient du chemin } \\
& \text { si lui donnez logis }{ }^{12}
\end{aligned}
$$

Nous restons sur notre faim.

Car cette musique de saint Jean-de-la-Croix, c'est la bonne nouvelle mystérieuse au milieu de la nuit : L'Époux est là ! La Vierge est là ! Où est votre lampe ? Ouvrez-lui ! (Mt, 25, 1-13).

C'est aussi, en filigrane, la musique des processions religieuses espagnoles, avec leurs chars, et leurs Christs et leurs Vierges, qui font station devant telle ou telle maison, selon les requêtes des habitants.

Peut-être pourrait-on traduire en transposant légèrement :

Du Verbe divin

La Vierge enceinte

Vient, depuis le chemin,

$\mathrm{Si}$ vous lui donnez logis.

Mais où est la musique? Et si l'on traduit : arrive sur votre chemin, où est le lien entre le Verbe divin et le chemin?

Tournons-nous vers Angelus Silesius, ce fascinant poète mystique allemand baroque, du XvII ${ }^{e}$ siècle. Par ses concetti, ces antithèses, ses chocs de musique et d'idées, il cerne aussi l'ineffable. Ses poèmes sont brefs, sonnets ou distiques, mais ils expriment des abîmes divins, ou abîmes de l'âme en Dieu, à la lumière de la théologie de l'inconnaissance divine, telle que la lui ont transmise les mystiques flamands et allemands du Moyen Âge, notamment Ruysbroek, Tauler at Suso.

Chacun de ses textes est une sorte de perle mystérieuse, noyée dans le soleil, et par là difficile à percevoir. D'où d'extrêmes difficultés de traduction.

12. Vida e Obras completas de San Juan de la Cruz, op. cit., p. 416. Lavaud, op. cit., p. 125. 
Il est le poète d'une sorte de dissolution des choses :

Entbildet mustu seyn

Entbilde dich mein Kind, so wirstu Gotte gleich :

Und bist in stiller Ruh dir selbst dein Himmelreich.

Il faut perdre toute forme.

Perds toute forme, mon enfant, tu deviendras semblable à Dieu ;

Et tu seras, dans une immobile quiétude, ton propre royaume des cieux ${ }^{13}$.

C'est l'obscur qui le conduit à la clarté, qui lui fait pressentir la lumière ; le moins qui lui fait entrevoir le plus et le tout.

Gott kennt man durch die Sonne.

Die Sonn ist nur ein Glast, und alles Licht ein schein :

Was muss doch für ein Blitz, Gott meine Sonne seyn!

On connaît Dieu par le soleil.

Le soleil n'est que miroitement; toute lumière, que reflet;

Quel éclair doit donc être Dieu, mon Soleil ${ }^{14}$ !

C'est le poète du raccourci :

Wie der Mund, so der Trank.

Die Hure Babylon trinkt Blutt, und trinkt den Todt :

O grosser unterscheid! Ich trinke Blutt und Gott.

Telle la bouche, telle la boisson.

La garce Babylone boit du sang, boit la mort :

$\mathrm{O}$ grande différence! Je bois du sang et Dieu ${ }^{\text {i5 }}$.

En deux vers, il fait jaillir le drame biblique des deux Cités, la Cité de Dieu et la Cité de Satan; la réalité et le symbolisme de Babylone - qui est le monde, ou le peuple, ou la caste sacerdotale, ou l'âme pécheresse, détournés de Dieu ou hostiles à Dieu —, Babylone, «Cité du néant» (Is 24, 10), ville des cruautés de toutes sortes, ivre du sang des saints (Ap 17); toute la théologie de l'Eucharistie, sacrement de nourriture, de sacrifice, sacrement eschatologique ; les leçons bibliques sur le vin, la vigne et la vendange.

Le traducteur doit sonder et contempler chaque mot. Voyons, par exemple, l'énigmatique poème :

Der da war, ist und kommen wird, in Apocal.

Der Vatter war zuvor, der Sohn ist noch zur Zeit,

Der heilge Geist wird seyn im Tag der Herrlichkeit.

Le traducteur écrit :

Celui qui était, qui est, qui viendra, (dans l'Apocalypse)

13. Angelus Silesius, Pèlerin Chérubinique (Cherubinischer Wandersmann), édition bilingue, traduction par Henri Plard, Aubier, 1946, Livre II, 53, p. 118 (Nous renvoyons à la page du texte allemand).

14. Id.,II, 156, p. 134

15. Id., I, 209, p. 94. 
Le Père fut avant, le Fils encore,

Le Saint-Esprit sera au jour de la Splendeur ${ }^{16}$.

Qu'est-ce à dire? Le Père n'est plus?

En vérité il s'agit ici d'une interprétation biblique, due à la pensée orthodoxe ou dissidente chrétienne, qui divise l'histoire de l'bumanité en trois temps súr terre : le Règne du Père, ou temps de la Loi ; le Règne du Fils, ou temps de la Grâce; le Règne - encore à venir - du Saint-Esprit, ou temps de la Gloire. Joachim de Flore en fut un des prophètes. Saint Grignion de Montfort aussi : "Le règne spécial de Dieu le Père a duré jusqu'au déluge et a été terminé par un déluge d'eau; le règne de Jésus-Christ a été terminé par un déluge de sang, mais votre règne, Esprit du Père et du Fils, continue à présent et sera terminó par un déluge de feu, d'amour et de justice. »Et il s'écrie : "Quand sera-ce que viendra ce déluge de feu du pur amour que vous devez allumer sur toute la terre d'une manière si douce et si véhémente que toutes les nations, les Turcs, les idolâtres et les Juifs même en brûleront et se convertiront ? Non est qui se abscondat a calore ejus (Ps 18,7) ${ }^{17}$. $\gg$ Cette jubilation, qui suit la Grande Tribulation, est un des grands thèmes de Marie des Vallées, la mystique de Coutance, fille spirituelle de saint Jean-Eudes. \& Elle pria Dieu « de donner des yeux aux aveugles», ce qui lui fut accordé pour le temps de la Conversion Générale, par cet arrêt prononcé par la bouche du Fils de Dieu : «Je condamne le Soleil à donner des yeux aux aveugles ${ }^{18}$.

Ne serait-il donc pas plus juste, et plus conforme à la tradition qui inspire Angelus Silesius, de traduire : le Saint-Esprit sera au jour de la Gloire... ou encore : au Temps de la Seigneurie.

Parfois Silesius est intraduisible.

$\mathrm{AB}$ ist schon genug.

Die Heyden plappern vil : wer Geistlich weiss zubetten, Der kan mit A und B getröst für Gott hintretten.

A B B A.

Ill joue avec les lettres du mot : Abba, qui, en araméen, signifie : Père.

Le traducteur a traduit :

$\mathrm{AB}$ suffit bien.

Les païens babillent fort : qui veut prier en spirituel peut tranquillement se présenter à Dieu avec $\mathrm{A}$ et $\mathrm{B}$. A B B A ${ }^{19}$.

Même si l'on transforme :

Les païens babillent fort; qui sait prier en esprit, est-on plus clair?

qu'il se présente, avec confiance, à Dieu, avec $\mathbf{A}$ ' et $B$,

16. Angelus Silesius, op. cit., Livre III, 215, p. 190.

17. Saint Louis-Grignion de Montfort, Euvres complètes, Seuil, 1966. Cf. Prière embrasé, Mémento, 16,17, p. 681 .

18. La Vie admirable et les révélations de Marie des Vallées, par Emile Dermenghem, Plon, 1926, p. 225.

19. Angelus Silesius, op. cit., Livre II, 77, p. 122. 
IV

Traduire un poète est donc une périlleuse acrobatie.

Et quand il s'agit d'un poète mystique, c'est presque se battre avec la quadrature du cercle.

«Le poème n'est accompli que s'il se fait chant, parole et musique en même temps », dit l'africain Sédar Senghor dans ses Éthiopiques ${ }^{20}$.

Il en est de même de la traduction.

Qui traduit un poème est appelé à jouer de toutes sortes d'instruments et toutes sortes de musiques :

- À l'échelle du vent...

- Qui pratiquent des brèches dans le sommet comme un soleil levant...

- Couleur de tous les jours...

- Qui soient à nos soifs une eau fraîche...

- Feux qui l'aveugle, et tonnerres au sourd... ${ }^{21}$

Le traducteur de poèmes poursuit-il une chimère?

Sur la difficulté de traduire les allusions ou les précisions religieuses, en poésie, nous pourrions encore citer deux exemples. Un poème de Yeats, THE SONG OF WANDERING AENGUS, se termine ainsi :

Though I am old with wandering

Through hollow lands and hilly lands,

I will find out where she has gone,

And kiss her lips and take her hands ;

And walk among long dappled grass,

And pluck till time and times are done

The silver apples of the moon,

The golden apples of the sun.

J. M. Rivet et M. P. Fouchet traduisent :

Bien que vieilli dans la vie vagabonde

Par plaines et collines, par monts et par vaux,

Je l'atteindrai dans sa fugue lointaine

Et presserai ses lèvres et prendrai ses mains ;

Et sans trêve irai au travers les grands prés miroitants

Et cueillerai jusqu'à la fin des temps et des temps

De la lune, les pommes d'argent

Du soleil les pommes d'or.

(Fontaine, op. cit., p. 520)

M. L. Cazamian propose, dans son anthologie bilingue, Yeats, Poèmes choisis (Aubier, 1954) :

Bien que j'ai vieilli en errant

A travers vallons et collines,

Je veux découvrir où elle est allée,

20. Léopold Sédar Senghor : Ethiopiques, Poèmes, Seuil, 1956.

21. Aragon, "Je ne connais pas cet homme», dans la Diane française, Seghers, 1945, p. 27. 
Baiser ses lèvres, lui prendre les mains ;

Marcher dans les herbes hautes et fleuries,

Et cueillir jusqu'à la fin des temps

Les pommes d'argent de la lune,

Les pommes d'or du soleil.

Il y a, dans le texte de Yeats, une distinction : time, times, qui n'est rendue par aucune des deux traductions. Il faut traduire ici : jusqu’à la fin du temps et des temps.

Cette distinction, l'Écriture Sainte la fait, en distinguant bien la fin du temps - l'entrée dans l'Éternité - de la Fin des Temps, ou fin des temps des hommes, des nations.

Jean Guitton remarque dans une étude sur la Phénoménologie mystique : «Si on rapproche l'expérience mystique chrétienne de la métaphysique du Temps, on peut noter que le mystique s'oriente dans deux axes d'à-venir, distincts et mystérieusement unis par l'existence : le futur et l'éternel, qui s'uniront lors de l'arrêt du temps, à la fin du temps, à la fin des temps, dans la Présence, dont le moment, le présent, l'instant sont des figures. Ainsi la mort sera «vaincue, passée et dépassée. Et, dans ses plus hauts états, sous des formes différentes ici plus anéanties (Bouddhisme et Plotin), là plus ardentes, plus super-conscientes (Christianisme, saint Augustin), ou encore plus synthétiques et plus conciliantes et oscillantes (saint Jean-de-la-Croix), le mystique anticipe sur l'au-delà, sur la vie après la mort» (Colloque sur les Visions mystiques, Paris, 17, 18 mars 1976, p. 18).

Un rien peut changer le tout. Angelus Silesius écrit :

Gott is mein Punct und Kreiss.

Gott ist mein mittelpunct wenn ich Ihn in mich schlisse :

Mein Umkreiss dann, wenn ich auss Lieb' in jhn zerflisse.

(Cherubinischer Wandersmann, drittes Buch, 148).

«Dieu est mon point et mon cercle, dit le traducteur. Dieu est mon centre quand je m'enferme en Lui ; et une circonférence, quand, par amour, je me fonds en Lui. » Il y a erreur grave. Le sens réel est : Dieu est mon centre quand je l'enferme en moi (quand je m'enferme en lui, d'une certaine manière il est mon centre, mais il est encore plus ma circonférence; et de toutes façons, le texte allemand porte : wenn ich Ihn in mich schlisse).

ROLAND MAISONNEUVE 